\title{
Surgical assessment of the insula. Part 2: validation of the Berger-Sanai zone classification system for predicting extent of glioma resection
}

\author{
Shawn L. Hervey-Jumper, MD, Jing Li, MD, Joseph A. Osorio, MD, PhD, Darryl Lau, MD, \\ Annette M. Molinaro, PhD, Arnau Benet, MD, and Mitchel S. Berger, MD \\ Department of Neurological Surgery, University of California, San Francisco, California
}

OBJECTIVE Though challenging, maximal safe resection of insular gliomas enhances overall and progression-free survival and deters malignant transformation. Previously published reports have shown that surgery can be performed with low morbidity. The authors previously described a Berger-Sanai zone classification system for insular gliomas. Using a subsequent dataset, they undertook this study to validate this zone classification system for predictability of extent of resection (EOR) in patients with insular gliomas.

METHODS The study population included adults who had undergone resection of WHO Grade II, III, or IV insular gliomas. In accordance with our prior published report, tumor location was classified according to the Berger-Sanai quadrant-style classification system into Zones I through IV. Interobserver variability was analyzed using a cohort of newly diagnosed insular gliomas and independent classification scores given by 3 neurosurgeons at various career stages. Glioma volumes were analyzed using FLAIR and T1-weighted contrast-enhanced MR images.

RESULTS One hundred twenty-nine procedures involving 114 consecutive patients were identified. The study population from the authors' previously published experience included 115 procedures involving 104 patients. Thus, the total experience included 244 procedures involving 218 patients with insular gliomas treated at the authors' institution. The most common presenting symptoms were seizure (68.2\%) and asymptomatic recurrence (17.8\%). WHO Grade II glioma histology was the most common (54.3\%), followed by Grades III (34.1\%) and IV (11.6\%). The median tumor volume was $48.5 \mathrm{~cm}^{3}$. The majority of insular gliomas were located in the anterior portion of the insula with $31.0 \%$ in Zone I, $10.9 \%$ in Zone IV, and $16.3 \%$ in Zones I+IV. The Berger-Sanai zone classification system was highly reliable, with a kappa coefficient of 0.857 . The median EOR for all zones was $85 \%$. Comparison of EOR between the current and prior series showed no change and Zone I gliomas continue to have the highest median EOR. Short- and long-term neurological complications remain low, and zone classification correlated with short-term complications, which were highest in Zone I and in Giant insular gliomas.

CONCLUSIONS The previously proposed Berger-Sanai classification system is highly reliable and predictive of insular glioma EOR and morbidity. http://thejns.org/doi/abs/10.3171/2015.4.JNS1521

KEY WORDS anaplastic astrocytoma; glioma; glioblastoma; low-grade glioma; insular glioma; oncology

I NSULAR gliomas remain a challenge to manage. Given the complexity of the insular lobe, its proximity to functionally significant areas, and its intimate relationship with middle cerebral and lenticulostriate artery branches, these tumors were often deemed too dangerous for surgical treatment. However, improvements in neuroanesthesia, microsurgical technique, and functional mapping have allowed greater access to these tumors with a low complication rate. Prior published reports suggest that aggressive resection of both low- and high-grade insular gliomas may be accomplished with an acceptable morbidity profile..$^{2,3,7-10,13,14,17}$ Maximal extent of resection (EOR) predicts superior overall and progression-free survival as well as improved seizure control., ${ }^{6,11,13}$ The majority of insular gliomas not only involve the insular lobe but can also infiltrate into portions of the frontal operculum and temporal lobe. Given their proximity to functional language and motor networks, the surgical approach may vary depending on the predominant component of the tumor within the insula. Recent publications have focused on the role of

ABBREVIATIONS EOR = extent of resection; FLAIR = fluid-attenuated inversion recovery; IDH = isocitrate dehydrogenase; WHO = World Health Organization. SUBMITTED January 5, 2015. ACCEPTED April 9, 2015.

INCLUDE WHEN CITING Published online September 4, 2015; DOI: 10.3171/2015.4.JNS1521. 
surgery to improve survival for patients with insular gliomas..$^{6,8,13-15}$ In our prior retrospective series, we analyzed perioperative outcomes after surgery for 115 consecutive insular gliomas focusing on morbidity and the effect of EOR on patient outcome and proposed an anatomical classification system for insular gliomas to help preoperatively predict the likely extent of tumor resection..$^{13}$ In this current study, we assign the previously described "Berger-Sanai" classification system to a new cohort of insular gliomas to determine the interobserver reliability among clinicians at different levels of clinical experience and expertise in order to validate our previous EOR predictions.

\section{Methods \\ Patient Selection}

Using a prospectively collected database of insular gliomas assigned to one of 4 previously described zones, ${ }^{13}$ we studied 114 consecutive patients undergoing a total 129 resections between September 2007 and April 2014. All procedures were performed by the study's senior author (M.S.B). Patients were all adults older than 18 years of age who had undergone surgery at the University of California, San Francisco. Perioperative patient parameters including zone classification based on preoperative fluid-attenuated inversion recovery (FLAIR) and T1-weighted postcontrast MR images, clinical presentation, handedness, age at diagnosis, immediate postoperative MRI (within 48 hours of surgery), and histopathology review (in accordance with World Health Organization [WHO] guidelines) were prospectively collected. Given substantial differences in their natural history, patients with WHO Grade I histology, those with multifocal glioma, and gliomatosis cerebri were excluded from analysis. All aspects of microsurgical tumor removal, including a description of the functional mapping, have been previously described in our prior publication on insular gliomas..$^{13}$ Approval for this study was granted by the University of California, San Francisco Committee on Human Research.

\section{Interobserver Reliability of Berger-Sanai Insular Glioma Classification System}

According to our prior published protocol, the insula was divided into 4 zones (Fig. 1). Along the horizontal plane in a sagittal view, the insula was seen to straddle the overlying sylvian fissure. This plane was intersected by a perpendicular line at the foramen of Monro. Tumor location was assigned to one or more of these zones. ${ }^{13}$ For tumors occupying more than 1 zone, this condition was denoted as such (e.g., Zones I+II). For cases in which the tumor occupied all 4 zones, these insular gliomas were defined as "Giant." Using this method, a total of 9 possible options existed for classification: Zones I, II, III, IV, I+II, I+IV, II+III, and III+IV and Giant.

To test agreement of insular zone assignment between clinicians, a subset of 80 cases of newly diagnosed insular glioma were independently scored by 3 examiners. With the goal of testing clinicians across a varied distribution of clinical experiences, 1 junior level neurosurgery resident (J.O.), 1 junior level neurosurgery faculty member (S.H.J.), and 1 senior neurosurgeon (M.S.B.) were chosen to participate. Participants were blinded to each other's score.
The kappa coefficient was used to determine the significance of this agreement. Interpretation of the kappa coefficient was performed in accordance to prior published reports in which 0 indicated agreement equivalent to chance, $0.01-0.20$ slight agreement, $0.21-0.40$ fair agreement, $0.41-0.60$ moderate agreement, $0.61-0.80$ substantial agreement, 0.81-0.99 almost perfect agreement, and a kappa coefficient of 1 indicated perfect agreement. ${ }^{5}$

\section{Patient Outcome Measurements}

Patients underwent sequential neurological examinations performed by 4 clinicians during the perioperative period: the senior attending neurosurgeon, a neurosurgical resident, a speech and language neurophysiologist, and the attending neuro-oncologist. Clinical examinations were performed preoperatively, every day during the postoperative period, and at each follow-up appointment by at least 1 of the above-mentioned clinicians (4-6 weeks and 3-6 months following surgery). Short-term neurological morbidity was defined as new-onset language or sensorimotor deficits within the first 3-5 postoperative days. Long-term neurological morbidity was defined as persistent dysfunction 90 days after surgical intervention. Our protocol for language function testing has been previously described. ${ }^{12}$ Differences between findings of the 4 examiners were adjudicated by accepting the results showing the greatest impairment if more than 1 examiner was involved at a given time point. MRI results were reviewed to confirm that the patient's symptoms were not a function of tumor progression at each time point. Malignant progression was defined as a change in histopathology of WHO Grade II or III tumors to higher-grade lesions on a subsequent procedure.

\section{Volumetric Analyses}

One author (J.L.) conducted volumetric measurement of pre- and postoperative imaging. Each measurement and calculation was examined for accuracy by another author (S.H.J.) and the primary surgeon (M.S.B.). Lowand high-grade tumors were volumetrically analyzed by measuring hyperintense regions on axial T2-weighted FLAIR MR images (low-grade gliomas) and T1-weighted contrast-enhanced MR images (high-grade gliomas). For each case, the tumor was segmented manually across all slices with region-of-interest analysis to compute pre- and postoperative volumes in cubic centimeters. The EOR was calculated as follows: [100 - (postoperative tumor volume/ preoperative tumor volume)*100], with $100 \%$ indicating gross total resection and $<100 \%$ representing subtotal resection. Determination of tumor volume and EOR was made without consideration of the clinical outcome.

\section{Statistical Analyses}

Descriptive statistics were calculated for all variables and stated as median (unless otherwise specified) for continuous variables and frequency of distribution for categorical variables. Cross-tabulations were generated, and the Wilcoxon signed-rank test (for continuous variables) and chi-square (for categorical variables) tests were used to compare distributions. The Fisher exact test was used if more than $80 \%$ of values were less than 5 . The kappa coefficient was used to determine strength of agreement be- 

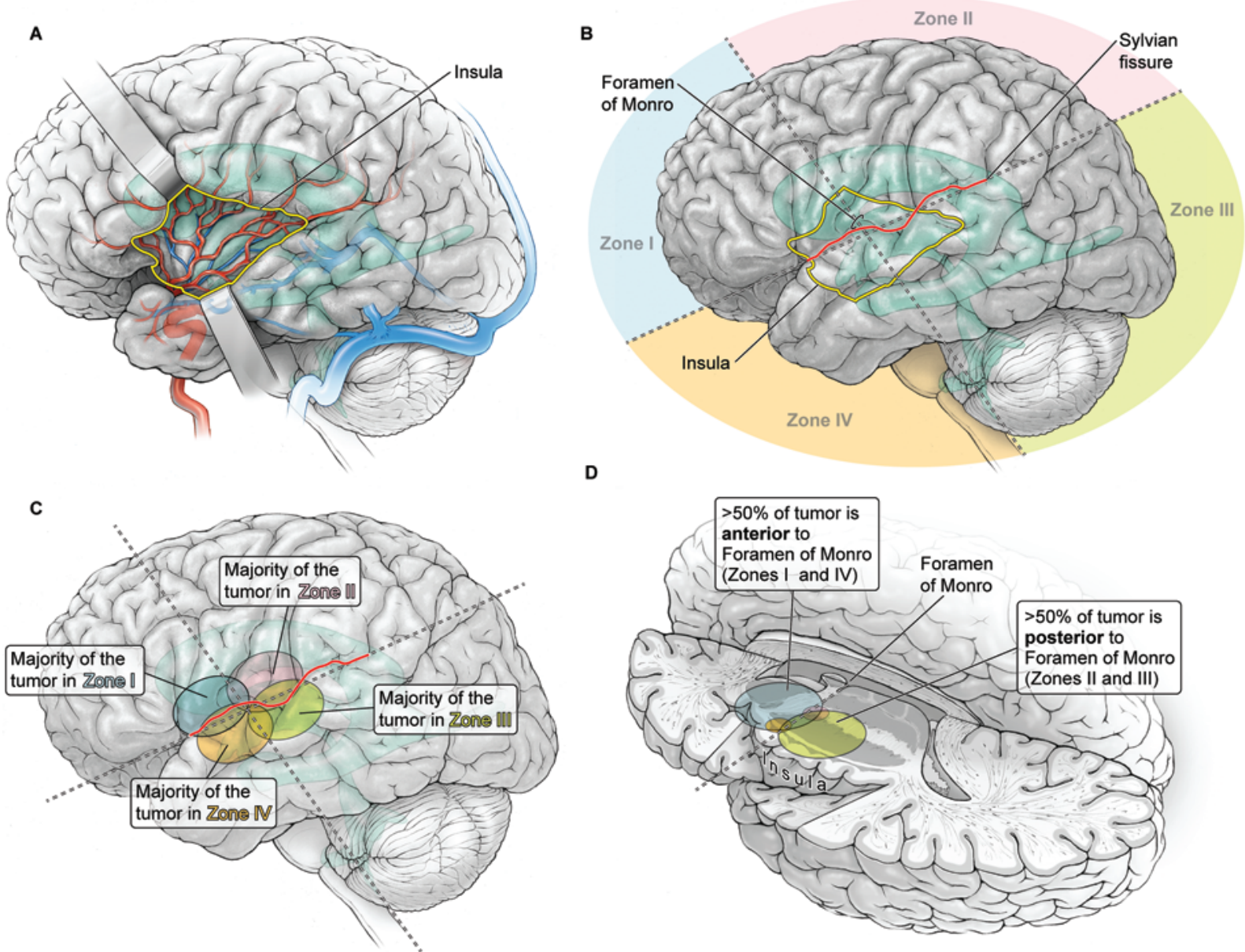

FIG. 1. Illustration showing insular surface with Berger-Sanai insular glioma classification system. A: The insula is covered by the frontoparietal and temporal opercula. B: Zones I to IV are divided along the sylvian fissure and a perpendicular plane crossing the foramen of Monro. C: Insular tumor location is determined by the position of the majority of the tumor mass. D: Axial illustration of Zones I and II located anterior to the foramen of Monro and Zones II and III located behind the foramen of Monro. Copyright Kenneth X. Probst. Published with permission.

tween clinicians. All p values were obtained from 2-sided tests, with statistical significance defined as $\mathrm{p}<0.05$. A biostatistician (A.M.) assisted with statistical analyses in this study, using JMP statistical software, version 10.0.2 (SAS Institute, Inc.).

\section{Results}

\section{Patient Demographics}

The study population included 114 consecutive patients for a total of 129 procedures representing 74 men and 55 women with a median age of 41 years (range 18-72 years) (Table 1). The study population from our previously published experience included 115 procedures involving 104 patients. As such, our total experience included 244 procedures involving 218 patients with insular glioma treated at our institution. Eighty $(62.0 \%)$ of the 129 procedures in the current experience represented primary craniotomies, whereas $49(38.0 \%)$ of the operations involved patients who had undergone at least 1 prior surgical procedure for the treatment of insular glioma. Sixty-eight $(52.7 \%)$ of the procedures were for the treatment of left-sided tumors, and there were 58 awake craniotomy procedures (45.0\%). Pa- tients most commonly presented with new-onset seizures (88 cases, $68.2 \%$ ), or asymptomatic tumor progression (23 cases, 17.8\%); other presentations included headaches (9 cases, $7.0 \%$ ), cognitive decline (3 cases, $2.3 \%$ ), motor deficits (3 cases, 2.3\%), and language deficits ( 1 case, $0.8 \%$ ). Insular gliomas were rarely discovered incidentally, representing $1.6 \%$ of cases ( 2 patients). In the 129 operations, the most common histological grade was WHO Grade II (70 tumors, 54.3\%), followed by WHO Grade III (44 tumors, 34.1\%), and WHO Grade IV (15 tumors, 11.6\%). The median tumor volume was $48.5 \mathrm{~cm}^{3}$ (range $0.11-245.7$ $\mathrm{cm}^{3}$ ). Fifteen percent of insular tumors (19) were confined entirely within the insula, while $85 \%$ of tumors were primarily based in the insula (i.e., $>75 \%$ ) with tumor extending beyond the insula to involve portions of the frontal, temporal, or parietal lobes. The median duration of clinical follow-up was 3.5 years (range 0.26-25.8 years) and, as part of planned adjuvant therapy, 107 patients (82.9\%) were treated with chemotherapy and $82(63.6 \%)$ underwent radiation therapy after surgery (decisions about use of adjuvant chemo-radiation were made on a case-by-case basis dependent on tumor recurrence, WHO grade, and histol- 
TABLE 1. Demographic and clinical characteristics of study patients who underwent surgery for treatment of insular glioma (129 procedures)

\begin{tabular}{|c|c|}
\hline Parameter & Value \\
\hline \multicolumn{2}{|l|}{ Age at diagnosis (yrs) } \\
\hline Median & 41 \\
\hline Range & $18-72$ \\
\hline \multicolumn{2}{|l|}{ Sex } \\
\hline Male & $74(57.4 \%)$ \\
\hline Female & $55(42.6 \%)$ \\
\hline \multicolumn{2}{|l|}{ Side of tumor } \\
\hline Left & $68(52.7 \%)$ \\
\hline Right & $61(47.3 \%)$ \\
\hline \multicolumn{2}{|l|}{ WHO tumor grade } \\
\hline II & $70(54.3 \%)$ \\
\hline III & $44(34.1 \%)$ \\
\hline IV & $15(11.6 \%)$ \\
\hline \multicolumn{2}{|l|}{ Tumor volume $\left(\mathrm{cm}^{3}\right)$} \\
\hline Median & 48.5 \\
\hline Range & $0.11-245.7$ \\
\hline \multicolumn{2}{|l|}{ Insular glioma location by zone } \\
\hline I & $40(31.0 \%)$ \\
\hline ॥ & $2(1.6 \%)$ \\
\hline III & $17(13.2 \%)$ \\
\hline IV & $14(10.9 \%)$ \\
\hline$|+| \mid$ & $4(3.1 \%)$ \\
\hline I+IV & $21(16.3 \%)$ \\
\hline$\|+\| \|$ & $7(5.4 \%)$ \\
\hline III+IV & $12(9.3 \%)$ \\
\hline Giant & $12(9.3 \%)$ \\
\hline \multicolumn{2}{|c|}{ Median insular glioma volume by zone $\left(\mathrm{cm}^{3}\right)$} \\
\hline I & 49.1 \\
\hline II & 11.4 \\
\hline III & 22 \\
\hline IV & 20.1 \\
\hline $1+\|$ & 72 \\
\hline I+IV & 52.3 \\
\hline$\|+\| \|$ & 63.8 \\
\hline III+IV & 41.4 \\
\hline Giant & 91.2 \\
\hline \multicolumn{2}{|l|}{ Handedness } \\
\hline Right & $126(97.7 \%)$ \\
\hline Left & $3(2.3 \%)$ \\
\hline \multicolumn{2}{|l|}{ Symptoms at presentation } \\
\hline Seizure & $88(68.2 \%)$ \\
\hline Cognitive decline & $3(2.3 \%)$ \\
\hline Headache & $9(7.0 \%)$ \\
\hline Incidental & $2(1.6 \%)$ \\
\hline Language deficit & $1(0.8 \%)$ \\
\hline Motor deficit & $3(2.3 \%)$ \\
\hline Asymptomatic recurrence & $23(17.8 \%)$ \\
\hline
\end{tabular}

(continued)
TABLE 1. Demographic and clinical characteristics of study patients who underwent surgery for treatment of insular glioma (129 procedures)* (continued)

\begin{tabular}{lc}
\hline \multicolumn{1}{c}{ Parameter } & Value \\
\hline Type of surgery & \\
\hline Motor mapping & $122(94.6 \%)$ \\
\hline Language mapping & $58(45.0 \%)$ \\
\hline Awake surgery & $58(45.0 \%)$ \\
\hline New & $80(62.0 \%)$ \\
\hline Reoperation & $49(38.0 \%)$ \\
\hline Adjuvant oncologic treatment & \\
\hline Patients with postoperative chemotherapy & $107(82.9 \%)$ \\
\hline Patients with postoperative radiation & $82(63.6 \%)$ \\
\hline Malignant transformation & $49(38.0 \%)$ \\
\hline Clinical follow-up (yrs) & 3.5 \\
\hline Median & $0.26-25.8$ \\
\hline Range & $1(0.8 \%)$ \\
\hline EOR & $19(14.7 \%)$ \\
\hline $0-40 \%$ & $58(45.0 \%)$ \\
\hline $41-69 \%$ & $51(39.5 \%)$ \\
\hline $70-89 \%$ & $85 \%$ \\
\hline$>90 \%$ & $40-100 \%$ \\
\hline Median & \\
\hline Range & \\
\hline Values indicate numbers of cases (by procedure) unless otherwise indicated
\end{tabular}

ogy). Histologically confirmed malignant transformation from WHO Grade II to WHO Grade III or from WHO Grade III to WHO Grade IV occurred in 49 cases (38.0\%). Tumor laterality was evenly distributed (Table 1).

\section{Distribution of Insular Glioma Location}

Based on our prior published report, ${ }^{13}$ insular gliomas were assigned to 1 of 4 zones (Table 1). The majority of insular gliomas were located in the anterior portion of the insula (anterior to the foramen of Monro), with $31.0 \%$ (40 cases) within the anterior-superior quadrant (Zone I), 10.9\% (14 cases) within the anterior inferior quadrant (Zone IV), and $16.3 \%$ (21 cases) within Zones I+IV (total of $58.2 \%$ of cases within the anterior insula). Twelve insular gliomas (9.3\%) were classified as Giant, occupying all 4 zones.

\section{Interobserver Reliability of Berger-Sanai Insular Glioma Classification System}

Interobserver reliability was tested using preoperative FLAIR or T1-weighted gadolinium-enhanced MR images obtained in 80 patients with new WHO Grade II, III, or IV insular gliomas. Three neurosurgeons with varying amounts of clinical experience scored each tumor's location according to our previously published zone classification criteria. ${ }^{13}$ Interobserver agreement was $89.1 \%$ (41 of 46 cases) for WHO Grade II gliomas, 84.0\% (21 of 25 cases) for WHO Grade III anaplastic gliomas, and $100 \%$ ( 9 of 9 cases) for WHO Grade IV gliomas. Overall observer agreement was $89 \%$. Interobserver reliability testing showed strong agreement with a kappa coefficient of 
0.857 (95\% CI 0.77-0.94; $\mathrm{p}<0.001$ ). There was no correlation between observer agreement and WHO grade ( $\mathrm{p}$ $=0.42$ ).

\section{Extent of Resection}

EOR was determined as follows: in 1 case $(0.8 \%)$, the EOR was less than or equal to $40 \%$; in 19 (14.7\%), it was between $41 \%$ and $69 \%$; in $58(45.0 \%)$, it was between $70 \%$ an $89 \%$; and in $51(39.5 \%$ ), it was greater than $90 \%$. The median EOR was $85 \%$ (range 40\%-100\%) across all zones (Table 1). Among cases of WHO Grade II glioma (70 cases), the median EOR was 81\%; in 55 cases (78.6\%), the EOR was greater than or equal to $70 \%$ (this included 32 cases [45.7\%] with 70\%-89\% resection and 23 [32.9\%] with $>90 \%$ resection). A total of 44 operations were for treatment of anaplastic astrocytoma (WHO Grade III), with a median EOR of $88 \%$. In 41 cases $(93.2 \%)$, the EOR was $70 \%$ or greater (this included 23 cases [52.3\%] with a $70 \%-89 \%$ resection and 18 [40.9\%] with a $>90 \%$ resection). Among cases of WHO Grade IV glioma (15 cases), the median EOR was $97 \%$, and 13 cases $(86.7 \%$ ) had an EOR greater than or equal to $70 \%$ (this included 3 cases [20.0\%] with $70 \%-89 \%$ resection and 10 [66.7\%] with > $90 \%$ resection). Zone II insular gliomas were the smallest, with a median tumor volume of $11.4 \mathrm{~cm}^{3}$, while Giant insular gliomas had a median volume of $91.2 \mathrm{~cm}^{3}$ (Table 1). The greatest EOR was accomplished in tumors located in Zones I (median EOR 90.1\%) and IV (median EOR 89.5\%), compared with Zones I+IV (median EOR 75\%) and Giant tumors (median EOR 80\%) $(\mathrm{p}=0.008)$ (Table 2). In our initial series, the smallest EOR was associated with Zone II tumors; however, with a greater willingness to maximize resections using either a transcortical surgical corridor through silent portions of the face motor cortex or splitting the posterior sylvian fissure, the median EOR increased to $83.5 \%$. There were no significant differences in median EOR between tumors across all zones in our 2 insular glioma series (Table 2).

In addition to zones predictive of EOR, there was also a positive correlation between tumor size and EOR. The median glioma volume was highest in Giant $\left(91.2 \mathrm{~cm}^{3}\right)$, Zone I+II $\left(72.0 \mathrm{~cm}^{3}\right)$, and Zone II+III $\left(63.8 \mathrm{~cm}^{3}\right)$ tumors. The smallest median tumor volumes were observed in
Zone II $\left(11.4 \mathrm{~cm}^{3}\right)$, Zone III $\left(22.0 \mathrm{~cm}^{3}\right)$, and Zone IV (20.1 $\mathrm{cm}^{3}$ ) tumors (median volumes for Zone I, Zone I+IV, and Zone III+IV tumors were $49.1 \mathrm{~cm}^{3}, 52.3 \mathrm{~cm}^{3}$, and 41.4 $\mathrm{cm}^{3}$, respectively) $(\mathrm{p}=0.002)$. In cases with $\mathrm{EOR}<40 \%$, the median tumor volume was $38 \mathrm{~cm}^{3}$ (mean $38 \mathrm{~cm}^{3}$ ); in those with EOR of $41 \%-69 \%$, the median tumor volume was $46 \mathrm{~cm}^{3}$ (mean $61 \mathrm{~cm}^{3}$ ); in those with EOR of 70\%$89 \%$, the median volume was $62 \mathrm{~cm}^{3}$ (mean $62 \mathrm{~cm}^{3}$ ); and in those with an EOR $>90 \%$, the median glioma volume was $25 \mathrm{~cm}^{3}$ (mean $\left.46 \mathrm{~cm}^{3}\right)(\mathrm{p}=0.0183)$. This suggests a more complete resection for smaller insular tumors.

\section{Insular Glioma Molecular Characteristics and Impact on EOR}

There is inconsistency in the literature regarding the rate of $1 \mathrm{p}$ and $19 \mathrm{q}$ chromosomal co-deletions in insular gliomas. ${ }^{4,16}$ In $60(46.5 \%)$ of the cases in our new series, the tumors were either WHO Grade II oligodendroglioma $(n=24)$, WHO Grade II oligoastrocytoma $(n=24)$, or WHO Grade III anaplastic oligodendroglioma or oligoastrocytoma $(n=12)$. Forty-three percent $(n=23)$ of WHO Grade II and III insular oligodendroglioma and oligoastrocytomas had $1 \mathrm{p} 19 \mathrm{q}$ co-deletions $(\mathrm{p}=0.04)$. Other previously published reports have suggested that isocitrate dehydrogenase (IDH) status confers a higher degree of resectability on WHO Grade III and IV gliomas. ${ }^{1}$ We therefore analyzed EOR focusing on insular gliomas that were positive for the IDH mutation (IDH+). There was no significant difference in the volume of IDH+ and nonmutated (IDH-) insular gliomas (IDH+ gliomas had a median tumor volume of $47.1 \mathrm{~cm}^{3}$, while IDH- insular gliomas had a median tumor volume of $28.9 \mathrm{~cm}^{3} ; \mathrm{p}=0.18$ ). Furthermore, IDH+ insular gliomas were evenly distributed across zones, with no statistically significant differences noted (Zone I, 36\%; Zone II, 2\%; Zone III, 13\%; Zone IV, 2\%; Zones I+II, 4\%; Zones I+IV, 19\%; Zones II+III, $6 \%$; Zones III+IV, 9\%; Giant, 4\%; $\mathrm{p}=0.13$ ). Among WHO Grade II insular gliomas, IDH+ tumors had a median EOR of $81 \%$, while IDH- WHO Grade II insular gliomas had a median EOR of $86 \%(p=0.62)$. WHO Grade III IDH+ insular gliomas had a median EOR of $79 \%$, while IDH- WHO Grade III insular gliomas had a median EOR of $88 \%$ ( $p=0.06)$. The median EOR for WHO Grade IV

TABLE 2. Summary of resected insular gliomas by zone $(n=244)$

\begin{tabular}{|c|c|c|c|c|c|c|}
\hline Zone & WHO Grade II & WHO Grade III & WHO Grade IV & $\begin{array}{c}\text { Median EOR, } \% \\
\text { (new series, } n=129 \text { ) }\end{array}$ & $\begin{array}{c}\text { Median EOR, } \% \\
\text { (combined series, } n=244)^{*}\end{array}$ & $p$ Value \\
\hline$I(n=80)$ & $50(62.5)$ & $21(26.3)$ & $9(11.3)$ & 90.1 & 92 & 0.47 \\
\hline II $(n=8)$ & $4(50.0)$ & $3(37.5)$ & $1(12.5)$ & 83.5 & 75.5 & 0.41 \\
\hline III $(n=23)$ & $11(47.8)$ & $8(34.8)$ & $4(17.4)$ & 88 & 89 & 0.75 \\
\hline IV $(n=20)$ & $9(45.0)$ & $8(40.0)$ & $3(15.0)$ & 89.5 & 89.2 & 0.79 \\
\hline $\mid+\|(n=8)$ & $5(62.5)$ & $2(25.0)$ & $1(12.5)$ & 86.5 & 78.9 & 0.35 \\
\hline $1+\operatorname{IV}(n=47)$ & $25(53.2)$ & $20(42.6)$ & $2(4.3)$ & 75 & 78 & 0.06 \\
\hline$\|+\| \|(n=16)$ & $13(81.3)$ & $2(12.5)$ & $1(6.3)$ & 85 & 84 & 0.77 \\
\hline III+IV $(n=16)$ & $8(50.0)$ & $6(37.5)$ & $2(12.5)$ & 82 & 83 & 0.61 \\
\hline Giant $(n=26)$ & $15(57.7)$ & $9(34.6)$ & $2(7.7)$ & 80 & 76.4 & 0.60 \\
\hline Totals & $140(57.4)$ & 79 (32.4) & $25(10.2)$ & & & \\
\hline
\end{tabular}

\footnotetext{
* Bold type is used to highlight the values for the combined series.
}

$\dagger$ For comparison of EOR between Sanai et al..$^{13}$ and the current series. 
IDH+ insular gliomas was 83\%, while IDH- WHO Grade IV insular gliomas had a median EOR of $95 \%(p=0.31)$.

\section{Morbidity Profile}

There were no deaths related to surgery in this series. The overall short-term complication rate was $26.4 \%$ (34 complications in 129 procedures) (Table 3). Short-term (within 3-5 days after surgery) neurological complications occurred most frequently after procedures involving Zone 1 and Giant insular gliomas. New motor neurological deficits excluding face motor weakness (within 3-5 days after surgery) occurred after $7.8 \%$ of the procedures (10 of 129). New face motor deficits occurred after $9.3 \%$ (12 of 129). Early postoperative language deficits occurred after $16.3 \%$ (21 of 129). At the 3-month follow-up visit, $99.2 \%$ of face motor deficits resolved. The overall long-term (90day) neurological deficit rate was 3.2\%. All but 1 language deficit resolved $(0.8 \%)$, while the long-term rate of motor disability also remained low at $1.6 \%(\mathrm{n}=2)$. These rates compared favorably with results from our prior retrospective series.

\section{Discussion}

The insula's proximity to middle cerebral artery vessels, primary motor and sensory areas, and the perisylvian language network makes accessing and resecting gliomas in this area challenging. Prior studies demonstrated that maximal resection of insular gliomas enhances overall and progression-free survival and improves seizure outcome. .,11,13 $^{1}$ Furthermore, surgery can be accomplished with a median EOR of $80 \%-82 \%$ and minimal morbidity of long-term language (0.8\%) and motor function (1.6\%). ${ }^{2,3,8,13,14}$ The significance of volumetric EOR on overall survival for patients with both low- and high-grade insular gliomas has been demonstrated in multiple previously published reports. ${ }^{6,13,14}$ Sanai et al. analyzed 115 procedures involving 104 patients with insular glioma and demonstrated a 5-year overall survival of $100 \%$ when the EOR was $90 \%$ and $84 \%$ for an EOR less than $90 \%$ in cases of low-grade glioma. In cases of high-grade glioma, the 2-year overall survival was $91 \%$ with an EOR of $90 \%$ and $75 \%$ when the EOR was less than $90 \% .^{13}$ This observation was later confirmed by Skrap et al., who found an overall survival of $92 \%$ for patients with an EOR greater than $90 \%$ and overall survival of $57 \%$ for those with an EOR less than $70 \% .{ }^{14}$ With respect to WHO Grade III gliomas, greater than $90 \%$ EOR has been shown to be associated with a 2-year overall survival of $78 \%$, in contrast with less than $90 \%$ EOR, which was associated with a 2-year overall survival of $19.6 \% .{ }^{14}$ Recently it has been further demonstrated that EOR greater than $90 \%$ for insular gliomas is predictive of a favorable postoperative seizure outcome. ${ }^{6}$

In our previously published retrospective series of patients with insular gliomas, we assessed postoperative morbidity and patient survival while describing an anatomical characterization system to help predict extent of tumor resection. In this study, we use the previously described classification scheme to 1) determine if it is robust when used by other surgeons and 2) determine if it continues to be predictive of extent of tumor resection. We found this system to be highly reliable with minimal variability
TABLE 3. Postoperative morbidity and complication rates in 129 procedures

\begin{tabular}{|c|c|c|c|c|}
\hline Variable & Short Term* & $\begin{array}{l}\text { Long } \\
\text { Term† }\end{array}$ & $\begin{array}{c}\text { Prior Series } \\
\text { Long-Term } \\
\text { Disability }\end{array}$ & $\begin{array}{c}p \\
\text { Valuef }\end{array}$ \\
\hline \multicolumn{5}{|l|}{ Morbidity } \\
\hline Language deficit & $21(16.3 \%)$ & $1(0.8 \%)$ & $1(1.0 \%)$ & \\
\hline Motor deficit & $10(7.8 \%)$ & $2(1.6 \%)$ & $2(1.7 \%)$ & \\
\hline Face motor deficit & $12(9.3 \%)$ & $1(0.8 \%)$ & $2(1.7 \%)$ & \\
\hline Complication rates & & & & 0.03 \\
\hline Zone I & $9(26 \%)$ & & & \\
\hline Zone II & $0(0 \%)$ & & & \\
\hline Zone III & $3(8.8 \%)$ & & & \\
\hline Zone IV & $1(3 \%)$ & & & \\
\hline Zones I+II & $2(5.9 \%)$ & & & \\
\hline Zones I+IV & $4(12 \%)$ & & & \\
\hline Zones II+III & $5(15 \%)$ & & & \\
\hline Zones III+IV & $4(12 \%)$ & & & \\
\hline Giant & $6(18 \%)$ & & & \\
\hline
\end{tabular}

between clinicians and highly predictive of the expected EOR across all zones.

Few insula-based gliomas are confined entirely within the insula (15\% in this series). Furthermore, depending on where within the insula a glioma is based, the surgical approach and anatomical considerations vary. For this reason, a common terminology is helpful when discussing individual lesions. Yaşargil et al. proposed a classification system based on whether the lesion is restricted to the insula (Type 3), part of the insula (Type 3A), or included in the adjacent operculum (Type 3B). ${ }^{18,19}$ In that classification system, insular lesions involving one or both of the paralimbic orbitofrontal and temporopolar areas are classified, respectively, as Type 5A or Type $5 \mathrm{~B} .{ }^{18,19} \mathrm{We}$ found that this classification system failed to address many of the anatomical features relevant to surgery for insular gliomas, such as proximity to potentially functional areas. Additionally, it is difficult to use this classification to preoperatively predict EOR. We therefore proposed a classification system based on an anatomical split of the insula along the sylvian fissure and foramen of Monro, thereby dividing it into 4 parts (Zones I-IV) using preoperative high-resolution MR images. ${ }^{13}$ This approach allowed us to consider and describe each insular tumor in relation to 1) the perisylvian language network (above and below the sylvian fissure in the dominant hemisphere), 2) primary sensory and motor areas (commonly for Zone I or II gliomas), 3) Heschl's gyrus (Zone III gliomas), and 4) middle cerebral artery branches (particularly lateral lenticulostriate branches found within the suprasylvian region of Zone I). It is critically important that a classification system have little variability between examiners. We tested this by asking 3 clinicians at varying stages in their careers to rate a cohort of insular gliomas, and found a strong correlation among examiners (kappa coefficient 0.857). 
We set out to determine if the zone classification was predictive of EOR based on the previously described EOR in our original series. We found no differences in EOR for any zone between these 2 series. In this current patient series, we therefore reconfirmed our prior observation that zone classification was predictive of EOR, with the highest EOR seen in Zone I and Zone IV tumors. We also showed that the zone classification appears to be predictive of short-term postoperative morbidity, with a modestly higher early complication rate seen in Giant and Zone I tumors, and lowest complication rates seen in Zone II and Zone IV tumors $(p=0.03)$. In this current series of patients, we identified a slightly higher rate of short-term face motor deficits, which likely corresponds to using a transcortical window of entry through silent portions of the face motor area for purposes of enhancing exposure. The rate of long-term morbidity continued to be minimal at $3.5 \%$ and virtually unchanged from our initial series (3.8\%). ${ }^{13}$

To our knowledge, the combined experience of this and our previously published report represents the largest series of insular glioma resections. The Berger-Sanai classification system is robust with little interobserver variability, and appears to validate our original description using this classification to predict EOR. Even so, there are study limitations that must be considered. Although there was little interobserver variability between clinicians with varying degrees of clinical experience and expertise, the investigation remained a single-institution study. The adoption of this classification system with external clinical validation is a topic of future study.

\section{Conclusions}

Maximal safe resection of insular gliomas continues to be associated with improved patient outcome and acceptable morbidity. Our previously proposed classification system is highly reliable and predictive of insular glioma EOR and perioperative morbidity.

\section{References}

1. Beiko J, Suki D, Hess KR, Fox BD, Cheung V, Cabral M, et al: IDH1 mutant malignant astrocytomas are more amenable to surgical resection and have a survival benefit associated with maximal surgical resection. Neuro Oncol 16:81-91, 2014

2. Duffau H: Surgery of low-grade gliomas: towards a 'functional neurooncology.' Curr Opin Oncol 21:543-549, 2009

3. Duffau H, Moritz-Gasser S, Gatignol P: Functional outcome after language mapping for insular World Health Organization Grade II gliomas in the dominant hemisphere: experience with 24 patients. Neurosurg Focus 27(2):E7, 2009

4. Gozé C, Rigau V, Gibert L, Maudelonde T, Duffau H: Lack of complete $1 \mathrm{p} 19 \mathrm{q}$ deletion in a consecutive series of 12 WHO grade II gliomas involving the insula: a marker of worse prognosis? J Neurooncol 91:1-5, 2009

5. Griessenauer CJ, Miller JH, Agee BS, Fisher WS III, Curé JK, Chapman PR, et al: Observer reliability of arteriovenous malformations grading scales using current imaging modalities. J Neurosurg 120:1179-1187, 2014

6. Ius T, Pauletto G, Isola M, Gregoraci G, Budai R, Lettieri $\mathrm{C}$, et al: Surgery for insular low-grade glioma: predictors of postoperative seizure outcome. J Neurosurg 120:12-23, 2014

7. Kim YH, Kim CY: Current surgical management of insular gliomas. Neurosurg Clin N Am 23:199-206, vii, 2012
8. Lang FF, Olansen NE, DeMonte F, Gokaslan ZL, Holland EC, Kalhorn C, et al: Surgical resection of intrinsic insular tumors: complication avoidance. J Neurosurg 95:638-650, 2001

9. Mehrkens JH, Kreth FW, Muacevic A, Ostertag CB: Long term course of WHO grade II astrocytomas of the Insula of Reil after I-125 interstitial irradiation. J Neurol 251:14551464, 2004

10. Moshel YA, Marcus JD, Parker EC, Kelly PJ: Resection of insular gliomas: the importance of lenticulostriate artery position. J Neurosurg 109:825-834, 2008

11. Pallud J, Audureau E, Blonski M, Sanai N, Bauchet L, Fontaine D, et al: Epileptic seizures in diffuse low-grade gliomas in adults. Brain 137:449-462, 2014

12. Sanai N, Mirzadeh Z, Berger MS: Functional outcome after language mapping for glioma resection. N Engl J Med 358:18-27, 2008

13. Sanai N, Polley MY, Berger MS: Insular glioma resection: assessment of patient morbidity, survival, and tumor progression. J Neurosurg 112:1-9, 2010

14. Skrap M, Mondani M, Tomasino B, Weis L, Budai R, Pauletto G, et al: Surgery of insular nonenhancing gliomas: volumetric analysis of tumoral resection, clinical outcome, and survival in a consecutive series of 66 cases. Neurosurgery 70:1081-1094, 2012

15. Vanaclocha V, Sáiz-Sapena N, García-Casasola C: Surgical treatment of insular gliomas. Acta Neurochir (Wien) 139:1126-1135, 1997

16. Wu A, Aldape K, Lang FF: High rate of deletion of chromosomes $1 p$ and $19 q$ in insular oligodendroglial tumors. J Neurooncol 99:57-64, 2010

17. Wu AS, Witgert ME, Lang FF, Xiao L, Bekele BN, Meyers $\mathrm{CA}$, et al: Neurocognitive function before and after surgery for insular gliomas. J Neurosurg 115:1115-1125, 2011

18. Yaşargil MG, von Ammon K, Cavazos E, Doczi T, Reeves JD, Roth P: Tumours of the limbic and paralimbic systems. Acta Neurochir (Wien) 118:40-52, 1992

19. Zentner J, Meyer B, Stangl A, Schramm J: Intrinsic tumors of the insula: a prospective surgical study of 30 patients. J Neurosurg 85:263-271, 1996

\section{Disclosure}

The authors report no conflict of interest concerning the materials or methods used in this study or the findings specified in this paper.

\section{Author Contributions}

Conception and design: Hervey-Jumper, Li, Berger. Acquisition of data: all authors. Analysis and interpretation of data: all authors. Drafting the article: Hervey-Jumper. Critically revising the article: Hervey-Jumper, Berger. Reviewed submitted version of manuscript: all authors. Approved the final version of the manuscript on behalf of all authors: Hervey-Jumper. Study supervision: Berger.

\section{Supplemental Information \\ Companion Paper}

Benet A, Hervey-Jumper SL, González Sánchez JJ, Lawton MT, Berger MS: Surgical assessment of the insula. Part 1: surgical anatomy and morphometric analysis of the transsylvian and transcortical approaches to the insula. DOI: $10.3171 / 2014.12$. JNS142182.

\section{Correspondence}

Shawn L. Hervey-Jumper, Department of Neurological Surgery, University of California, San Francisco, 505 Parnassus Ave., M779, San Francisco, CA 94143. email: herveyju@umich.edu. 